PROCEEDINGS OF THE

AMERICAN MATHEMATICAL SOCIETY

Volume 126, Number 10, October 1998, Pages 3097-3108

S 0002-9939(98)04474-8

\title{
A NOTE ON HARMONIC FORMS ON COMPLETE MANIFOLDS
}

\author{
LUEN-FAI TAM
}

(Communicated by Peter Li)

\begin{abstract}
In this note, we will prove that under certain conditions, the space of polynomial growth harmonic functions and harmonic forms with a fixed growth rate on manifolds which are asymptotically nonnegatively curved is finite dimensional. This is a partial generalization of the works of $\mathrm{Li}$ and Colding-Minicozzi. We will also give an explicit estimate for the dimension in case the manifold is a complete surface of finite total curvature. This is a generalization to harmonic forms of the work of $\mathrm{Li}$ and the author.
\end{abstract}

\section{INTRODUCTION}

After the work $[Y]$, it was conjectured by Yau that the space of harmonic functions of polynomial growth of degree at most $d$ on a complete noncompact manifold with nonnegative Ricci curvature is finite dimensional. This conjecture has stimulated many interesting works; see [L 2] for details. Finally, the conjecture was proved to be true in [L-T 1] for the case of linear growth, and by Colding and Minicozzi for the general case in a series of papers [C-M 1]-[C-M 6]. In fact, they prove that the the conjecture is also true if we only assume the manifold satisfy volume doubling and that Poincaré inequality holds on the manifold. It is well-known that these two properties are satisfied by manifolds with nonnegative Ricci curvature. In [L 1], Li gives a very simple proof of a more general result. He proves that on a complete manifold $M$ satisfying volume doubling and on which mean value inequality for positive subharmonic functions holds, then the space of harmonic functions of polynomial growth of degree at most $d$ is finite dimensional. Note that if a manifold satisfies volume doubling and that Poincaré inequality holds on the manifold then mean value inequality will hold on such a manifold $[\mathrm{G}, \mathrm{SC}]$, but the converse is not true [L 1]. In [C-M 7], simple proofs of the main results in their previous works [C-M 1]-[C-M 6] are also given. Sharp estimates on the dimension are obtained under various assumptions; see [C-M 6], [C-M 7] and in particular [L 1]. Using similar methods, results on harmonic sections can also be obtained. For example, in [L 1], Li proves that on a complete noncompact manifold with nonnegative curvature operator, then the space of harmonic forms with polynomial growth of order at most $d$ is finite dimensional, and sharp bound on the dimension is also obtained; see also [C-M 7].

Received by the editors February 19, 1997.

1991 Mathematics Subject Classification. Primary 58E20.

Research partially supported an Earmarked grant of Hong Kong.

(c)1998 American Mathematical Society 
It is proved in [C-M 7] that if a manifold has Ricci curvature decay at least quadratically to zero near infinity, and satisfies a volume comparison property introduced in [L-T 5] on the whole manifold, then it will satisfy volume doubling and the mean value inequality for positive subharmonic function also holds. One can apply the main theorem in [L 1] to such manifolds. However, if we assume only that the Ricci curvature of $M$ is nonnegative outside a compact set, then $M$ may not satisfy the above mentioned properties. In this direction, it is proved by Wang [W] that if $M$ has nonnegative Ricci curvature outside a compact set with finite first Betti number, then the space of linear growth harmonic function is of finite dimension, and the dimension can be estimated in terms of geometric data. By introducing a similar inner product as in $[\mathrm{W}]$, in this note, we will prove that if $M$ is a complete noncompact manifold with nonnegative Ricci curvature outside a compact set, and if each unbounded component of $M \backslash D$, where $D$ is a bounded domain, satisfies a certain kind of volume comparison property, then the space of polynomial growth harmonic functions of degree at most $d$ is again finite dimensional. A sharp estimate can also be obtained. This class of manifolds include manifolds with nonnegative sectional curvature outside a compact set; see [L-T 5]. If we further assume that the curvature operator is nonnegative outside a compact set, then similar results are true for harmonic forms. In fact, the curvature assumptions can be relaxed further as in [W]. We should emphasis that the arguments are more or less the same as in [L 1] together with some observations.

In [L-T 2], spaces of polynomial growth harmonic functions on a complete noncompact surface with finite total curvature have been studied thoroughly. Lower and upper bounds for the dimensions of those spaces are given explicitly. Hence we have the same bounds for spaces of polynomial growth harmonic two forms. In the second part of this note, we will fill the gap and give an explicit upper bound for the polynomial growth harmonic one forms on a complete surface with finite total curvature.

The author would like to thank Peter Li for many useful discussions.

\section{Dimension estimates OF HARMOniC FORMS}

Let $M$ be a complete noncompact manifold of dimension $n$, and $p \in M$ be a fixed point. Let $D$ be a compact set, and $E$ be an unbounded component of $M \backslash D$. We call $E$ an end of $M$ with respect to $D$. We say that $E$ satisfies (VC) if there exists a constant $\zeta>0$, such that

$$
V_{E}(r) \leq \zeta V_{x}\left(\frac{r}{2}\right)
$$

for all $x \in \partial B_{E}(r)$ and for $r$ large enough. Here we use $B_{E}(r)$ to denote $B_{p}(r) \cap E$, $\partial B_{E}(r)=\partial B(r) \cap E, V_{E}(r)$ is the volume of $B_{E}(r)$ and $A_{E}(r)$ is the area of $\partial B_{E}(r)$.

Let $\mathfrak{V}$ be a rank $m$ vector bundle over $M$ with a metric. Let $D$ be a compact set of $M$, and let $E_{1}, \ldots, E_{L}$ be the ends of $M$ with respect to $D$. For $r>r_{0}>0$, with $B_{p}\left(r_{0}\right) \supset D$, we define a positive semidefinite symmetric bilinear form $S_{r, r_{0}}$ on the space of sections $\Gamma(\mathfrak{V})$ of $\mathfrak{V}$ by

$$
S_{r, r_{0}}(u, v)=V_{p}^{-1}\left(2 r_{0}\right) \int_{B_{p}\left(2 r_{0}\right)}\langle u, v\rangle+\sum_{l=1}^{L} V_{E_{l}}^{-1}(r) \int_{B_{E_{l}}(r)}\langle u, v\rangle
$$

for $u, v \in \Gamma(\mathfrak{V})$. In practice, $S_{r, r_{0}}$ is always positive definite, and $\left(\mathfrak{V}, S_{r, r_{0}}\right)$ is an inner product space. 
Lemma 1.1. Let $M^{n}$ be a complete manifold such that $\operatorname{Ric}(x) \geq-K(1+r(x))^{-2}$, for some constant $K$, where $r(x)$ is the distance of $x$ from $p$. Let $E_{1}, \ldots, E_{L}$ be the ends of $M$ with respect to $B_{p}(1)$. Suppose each $E_{l}$ satisfies $(\mathbf{V C})$. Let $\mathcal{K}$ be a $k$-dimensional subspace of $\Gamma(\mathfrak{V})$ with the property that

$$
\Delta|u|^{2} \geq 0
$$

outside $B_{p}(1)$ and

$$
\Delta|u|^{2} \geq-a|u|^{2}
$$

in $B_{p}(1)$ for some $a \geq 0$ for all $u \in \mathcal{K}$. Then there is a constant $C$ depending only on $n, K, \zeta, a, L, m$ such that if $0<\epsilon<\frac{1}{4}$, and $\delta>0$, and if $\left\{u_{1}, \ldots, u_{k}\right\}$ is an orthonormal basis for $\mathcal{K}$ with respect to $S_{(1+\epsilon) r, 1}$, then

$$
\sum_{i=1}^{k} S_{r, 1}\left(u_{i}, u_{i}\right) \leq C \epsilon^{-n-\delta+1}
$$

for all $r$ large enough.

Proof. Let $E=E_{l}$ be one of the ends of $M$ with respect to $B_{p}(1)$. Since $E$ satisfies $(\mathrm{VC})$, there is a constant $\zeta$ such that for all $r>2$ and for all $x \in \partial B_{E}(r)$,

$$
V_{E}(r) \leq \zeta V_{x}\left(\frac{r}{2}\right)
$$

By the assumption on $M$, and volume comparison, see [B-C], there is a constant $C_{1}$ and $\nu>0$ depending only on $n$ and $K$, such that for all $r^{\prime}>r>2$,

$$
V_{E}\left(r^{\prime}\right) \leq\left(\frac{r^{\prime}}{r}\right)^{\nu} V_{E}(r)
$$

and

$$
r A_{E}(r) \leq C_{1} V_{E}(r) .
$$

Moreover, given any $\delta>0$, there is $r_{1}>2$ such that for all $x \in \partial B_{E}(R)$, with $R>r_{1}$, and for all $\frac{2}{3} R>r^{\prime}>r>0$,

$$
V_{x}\left(r^{\prime}\right) \leq\left(\frac{r^{\prime}}{r}\right)^{n+\delta} V_{x}(r)
$$

Let $r>\frac{4}{3} r_{1}$, and let $\left\{u_{1}, \ldots, u_{k}\right\}$ be an orthonormal basis for $\left(\mathcal{K}, S_{(1+\epsilon) r, 1}\right)$. Following [L 1], let $F(x)=\sum_{i=1}^{k}\left|u_{i}\right|^{2}(x)$. Then $F$ is subharmonic in $E$. For any $0<\epsilon<\frac{1}{4}$, and for $\frac{3}{4} r<\rho<r$, let $x_{0} \in \partial B_{E}(\rho)$ be such that

$$
F\left(x_{0}\right)=\sup _{\partial B_{E}(\rho)} F
$$

As in [L 1], one can find an orthogonal transformation $A$ of $\left(\mathcal{K}, S_{(1+\epsilon) r, 1}\right)$, such that if $v_{i}=A u_{i}$, then $v_{i}\left(x_{0}\right)=0$ for $i \geq m+1$. Note that $v_{i}=\sum_{j} a_{j i} u_{j}$ for some $k \times k$ orthogonal matrix $\left(a_{i j}\right)$. Hence $F(x)=\sum_{i=1}^{k}\left|v_{i}\right|^{2}(x)$, and $F(x) \leq \sum_{i=1}^{m}\left|v_{i}\right|^{2}\left(x_{0}\right)$ for $x \in \partial B_{E}(\rho)$. Observe that since $\rho>\frac{3}{4} r$ and $\epsilon<\frac{1}{4}$, we have $(1+\epsilon) r-\rho<\frac{1}{2} r<$ $\frac{2}{3} \rho$. Hence by the mean value inequality of $[\mathrm{L}-\mathrm{S}]$ and the assumption on the Ricci 
curvature of $M$, there is a constant $C_{2}>0$ depending only on $n, K$ such that

$$
\begin{aligned}
\sup _{\partial B_{E}(\rho)} F & =F\left(x_{0}\right) \\
& =\sum_{i=1}^{m}\left|v_{i}\right|^{2}\left(x_{0}\right) \\
& \leq C_{2} V_{x_{0}}^{-1}((1+\epsilon) r-\rho) \sum_{i=1}^{m} \int_{B_{x_{0}}((1+\epsilon) r-\rho)}\left|v_{i}\right|^{2} \\
& \leq C_{2} V_{x_{0}}^{-1}((1+\epsilon) r-\rho) \sum_{i=1}^{m} \int_{B_{E}((1+\epsilon) r)}\left|v_{i}\right|^{2} \\
& \leq m C_{2} V_{x_{0}}^{-1}((1+\epsilon) r-\rho) V_{E}((1+\epsilon) r)
\end{aligned}
$$

where we have used the fact that $\left\{v_{1}, \ldots, v_{k}\right\}$ is orthonormal with respect to $S_{(1+\epsilon) r, r_{0}}$, so that

$$
\begin{aligned}
\int_{B_{E}((1+\epsilon) r)}\left|v_{i}\right|^{2} & \leq V_{E}((1+\epsilon) r) S_{(1+\epsilon) r, 1}\left(v_{i}, v_{i}\right) \\
& =V_{E}((1+\epsilon) r) .
\end{aligned}
$$

By (1.5)

$$
V_{x_{0}}^{-1}((1+\epsilon) r-\rho) \leq\left(\frac{\frac{1}{2} r}{(1+\epsilon) r-\rho)}\right)^{n+\delta} V_{x_{0}}^{-1}\left(\frac{1}{2} r\right) .
$$

By (1.2), we have

$$
V_{x_{0}}^{-1}((1+\epsilon) r-\rho) \leq \zeta \cdot\left(\frac{\frac{1}{2} r}{(1+\epsilon) r-\rho}\right)^{n+\delta}\left(V_{E}(r)\right)^{-1}
$$

Hence using (1.3), (1.6) implies that

$$
\begin{aligned}
\sup _{\partial B_{E}(\rho)} F & \leq 2^{-n-\delta} m C_{2} \zeta V_{E}((1+\epsilon) r) V_{E}^{-1}(r)\left(1+\epsilon-\frac{\rho}{r}\right)^{-n-\delta} \\
& \leq 2^{-n-\delta} m C_{2} \zeta(1+\epsilon)^{\nu}\left(1+\epsilon-\frac{\rho}{r}\right)^{-n-\delta}
\end{aligned}
$$

Hence

$$
\begin{aligned}
\int_{B_{E}(r) \backslash B_{E}\left(\frac{3}{4} r\right)} F & =\int_{\frac{3}{4} r}^{r} \int_{\partial B_{E}(\rho)} F d \rho \\
& \leq \int_{\frac{3}{4} r}^{r} A_{E}(\rho)\left(\sup _{\partial B_{E}(\rho)} F\right) d \rho \\
& \leq 2^{-n-\delta} m C_{2} \zeta(1+\epsilon)^{\nu} \int_{\frac{3}{4} r}^{r} A_{E}(\rho) f(\rho) d \rho
\end{aligned}
$$


where $f(\rho)=(1+\epsilon-\rho / r)^{-n-\delta}$. Using (1.4), we have

$$
\begin{aligned}
\int_{\frac{3}{4} r}^{r} A_{E}(\rho) f(\rho) d \rho & \leq C_{1} \int_{\frac{3}{4} r}^{r} \frac{V_{E}(\rho)}{\rho} f(\rho) d \rho \\
& \leq \frac{4 C_{1}}{3} \frac{V_{E}(r)}{r} \int_{\frac{3}{4} r}^{r} f(\rho) d \rho \\
& \leq \frac{4 C_{1}}{3(n+\delta-1)} V_{E}(r) \epsilon^{-n-\delta+1}
\end{aligned}
$$

Hence

$$
\int_{B_{E}(r) \backslash B_{E}\left(\frac{3}{4} r\right)} F \leq C_{3} \epsilon^{-n-\delta+1} V_{E}(r)
$$

for some constant $C_{3}$ depending only on $n, K, \zeta, m$. Since $F$ is subharmonic in $M \backslash B_{p}(1)$,

$$
\sup _{B_{E}\left(\frac{3}{4} r\right)} F \leq \max \left\{\sup _{\partial B_{E}\left(\frac{3}{4} r\right)} F, \sup _{B_{p}(1)} F\right\}
$$

By (1.7), take $\rho=\frac{3}{4} r$, we have

$$
\sup _{\partial B_{E}\left(\frac{3}{4} r\right)} F \leq C_{5}
$$

for some constant $C_{5}$ depending only on $n, K, \zeta, m$. Since $\Delta F \geq-a F$ in $B_{p}(1)$, using the mean value inequality in [L-T 3] and arguing as before, we have

$$
\sup _{B_{p}(1)} F \leq C_{6}
$$

where $C_{6}$ depends only on $a, n, K, m$. Combining this with (1.9) and (1.10), we have

$$
\sup _{B_{E}\left(\frac{3}{4} r\right)} F \leq C_{7}
$$

where $C_{7}=\max \left\{C_{5}, C_{6}\right\}$. Hence

$$
\int_{B_{E}\left(\frac{3}{4} r\right)} F \leq C_{7} V_{E}(r) .
$$

By (1.8) and (1.12), using the fact the $0<\epsilon<\frac{1}{4}$, we have

$$
\int_{B_{E}(r)} F \leq C_{8} \epsilon^{-n-\delta+1} V_{E}(r) .
$$

The inequality is true for each end $E$ of $M$ with respect to $B_{p}(1)$. Hence

$$
\sum_{l=1}^{L} \sum_{i=1}^{k} V_{E_{l}}^{-1}(r) \int_{B_{E_{l}}(r)}\left|u_{i}\right|^{2} \leq L C_{8} \epsilon^{-n-\delta+1} .
$$

On the other hand, if $r$ is large enough so that all bounded components of $M \backslash B_{p}(1)$ are contained in $B_{p}\left(\frac{3}{4} r\right)$, we have

$$
\begin{aligned}
\sup _{B_{p}(2)} F & =\max \left\{\sup _{B_{p}(2) \backslash B_{p}(1)} F, \sup _{B_{p}(1)} F\right\} \\
& \leq \max \left\{\sup _{B_{p}\left(\frac{3}{4} r\right) \backslash B_{p}(1)} F, \sup _{B_{p}(1)} F\right\} \\
& \leq C_{7} .
\end{aligned}
$$


Hence

$$
V_{p}^{-1}(2) \sum_{i=1}^{k} \int_{B_{p}(2)}\left|u_{i}\right|^{2} \leq C_{7} .
$$

Combining this with (1.14), the result follows.

Suppose that the Ricci curvature of $M^{n}$ satisfies $\operatorname{Ric}(x) \geq-\lambda(r(x))$ where $\lambda$ : $[0, \infty) \rightarrow[0, \infty)$ is a nonincreasing function such that $\int_{0}^{\infty} r^{n-1} \lambda(r) d r<\infty$. In this case $M$ has finitely many ends [L-T 4]; that is, the number of unbounded components of $M \backslash D$ is bounded from above uniformly for all compact sets $D$. Assume each end of $M$ with respect to $B_{p}(1)$ satisfies (VC). This implies that each end of $M$ with respect to $B_{p}(r)$ also satisfies (VC) with the same constant $\zeta$ for all $r>1$. By modifying the proof of Lemma 1.1, we have the following generalization:

Lemma 1.2. Let $M^{n}$ be as above with $n \geq 3$. Let $\mathfrak{V}$ be a rank $m$ vector bundle over $M$ with a metric. Let $\mathcal{K}$ be a $k$-dimensional subspace of $\Gamma(\mathfrak{V})$, with the property that

$$
\Delta|u|^{2}(x) \geq-a \lambda(r(x))|u|^{2}(x)
$$

for all $u \in \mathcal{K}$. Then there is an $r_{0}>0$ depending only on $n, \lambda, a, M$, and $a$ constant $C$ depending also on the number $L$ of unbounded components of $M \backslash B_{p}\left(r_{0}\right)$, such that if $0<\epsilon<\frac{1}{4}$, and $\delta>0$, and if $\left\{u_{1}, \ldots, u_{k}\right\}$ is an orthonormal basis of $\left(\mathcal{K}, S_{(1+\epsilon) r, r_{0}}\right)$, then

$$
\sum_{i=1}^{k} S_{r, r_{0}}\left(u_{i}, u_{i}\right) \leq C \epsilon^{-n-\delta+1}
$$

for $r$ large enough. Here for any $r, S_{r, r_{0}}$ is defined as in (1.1) with $D=B_{p}\left(r_{0}\right)$.

Proof. By the assumption on $\lambda$ we have $\lambda(r)=o\left(r^{-n+1}\right)$. As in [L-T 4, p.378], there is a function $g>0$ defined on $M$ such that

$$
\Delta g(x) \geq a \lambda(r(x)),
$$

in the sense of distribution on $M \backslash\{p\}$, and $g(x) \rightarrow 0$ as $x \rightarrow \infty$. Choose $r_{0}>$ large enough so that $g \leq \frac{1}{2}$ on $M \backslash B_{p}\left(r_{0}\right)$. Let $r>2 r_{0}$, and let $\left\{u_{1}, \ldots, u_{k}\right\}$ be an orthonormal basis for $\left(\mathcal{K}, S_{(1+\epsilon) r, r_{0}}\right)$. Let $F=\sum_{i=1}^{k}\left|u_{i}\right|^{2}$ as before. Let $E$ be an end of $M$ with respect to $B_{p}\left(r_{0}\right)$ and let $b=\sup _{B_{E}\left(\frac{3}{4} r\right)} F$. Then in $B_{E}\left(\frac{3}{4} r\right)$

$$
\begin{aligned}
\Delta\left(b^{-1} F\right) & \geq-a \lambda \cdot\left(b^{-1} F\right) \\
& \geq-a \lambda .
\end{aligned}
$$

By (1.15), we have

$$
\Delta\left(g+b^{-1} F\right) \geq 0
$$

in $B_{E}\left(\frac{3}{4} r\right)$. Hence

$$
\sup _{B_{E}\left(\frac{3}{4} r\right)}\left(g+b^{-1} F\right) \leq \max \left\{\sup _{\partial B_{p}\left(r_{0}\right)}\left(g+b^{-1} F\right), \sup _{\partial B_{p}\left(\frac{3}{4} r\right) \cap E}\left(g+b^{-1} F\right)\right\} .
$$

However, $g \leq \frac{1}{2}$ outside $B_{p}\left(r_{0}\right)$, hence

$$
1 \leq \frac{1}{2}+\max \left\{\sup _{\partial B_{p}\left(r_{0}\right)} b^{-1} F, \sup _{\partial B_{p}\left(\frac{3}{4} r\right) \cap E} b^{-1} F\right\},
$$


and so

$$
\sup _{B_{E}\left(\frac{3}{4} r\right)} F \leq 2 \max \left\{\sup _{\partial B_{p}\left(r_{0}\right)} F, \sup _{\partial B_{p}\left(\frac{3}{4} r\right) \cap E} F\right\} .
$$

Using (1.17), one can proceed as in the proof of Lemma 1.1 to get the result.

Lemma 1.3. Let $M$ be a complete noncompact manifold and let $p \in M$ be a fixed point. Let $\mathfrak{V}$ be a vector bundle of rank $m$ over $M$ with a metric. Given $r_{0}>0$, let $E_{1}, \ldots, E_{L}$ be the unbounded components of $M \backslash B_{p}\left(r_{0}\right)$. Assume each $S_{r, r_{0}}$ is an inner product for $r>r_{0}$, where $S_{r, r_{0}}$ is defined as in (1.1) with $D=B_{p}\left(r_{0}\right)$. Let $\mathcal{K}$ be a $k$-dimensional subspace of $\mathfrak{V}$ with polynomial growth of degree at most $d$. Then for all $\beta>1$ and $r_{1}>r_{0}$ there is $r>r_{1}$ such that if $\left\{u_{1}, \ldots, u_{k}\right\}$ is an orthonormal basis for $\left(\mathcal{K}, S_{\beta r, r_{0}}\right)$ then

$$
\sum_{i=1}^{k} S_{r, r_{0}}\left(u_{i}, u_{i}\right) \geq k \beta^{-(2 d+1)} .
$$

Proof. Following exactly as in [L 1], let us denote $S_{r, r_{0}}$ by $S_{r}$, the trace of $S_{r}$ with respect to $S_{r^{\prime}}$ by $\operatorname{tr}_{r^{\prime}} S_{r}$, and the determinant of $S_{r}$ with respect to $S_{r^{\prime}}$ by $\operatorname{det}_{r^{\prime}} S_{r}$. Suppose the theorem is not true; then for some $\beta>1$, and $r_{1}>r_{0}$ such that for all $r>r_{1}$

$$
\operatorname{tr}_{\beta r} S_{r}<k \beta^{-(2 d+1)}
$$

Since

$$
\left(\operatorname{det}_{\beta r} S_{r}\right)^{\frac{1}{k}} \leq k^{-1} \operatorname{tr}_{\beta r} S_{r}
$$

we have

$$
\operatorname{det}_{\beta r} S_{r} \leq \beta^{-k(2 d+1)} .
$$

Let $r=r_{1}+1$ and iterating we have for all integers $t \geq 1$,

$$
\operatorname{det}_{\beta^{t} r} S_{r} \leq \beta^{-t k(2 d+1)} .
$$

Now choose a fixed orthonormal basis $\left\{u_{1}, \ldots, u_{k}\right\}$ of $\mathcal{K}$, with respect to $S_{r}$; then

$$
\begin{aligned}
S_{\beta^{t} r}\left(u_{i}, u_{j}\right) & =V_{p}^{-1}\left(2 r_{0}\right) \int_{B_{p}\left(2 r_{0}\right)}\left\langle u_{i}, u_{j}\right\rangle+\sum_{l=1}^{L} V_{E_{l}}^{-1}\left(\beta^{t} r\right) \int_{B_{E_{l}}\left(\beta^{t} r\right)}\left\langle u_{i}, u_{j}\right\rangle \\
& \leq C_{1}\left(1+\beta^{t} r\right)^{2 d}
\end{aligned}
$$

for some constant $C_{1}$ independent of $t$. Hence

$$
\operatorname{det}_{r} S_{\beta^{t} r} \leq\left(C_{1}\right)^{k} k !\left(1+\beta^{t} r\right)^{2 d k} .
$$

Hence

$$
\left(C_{1}\right)^{-k}(k !)^{-1}\left(1+\beta^{t} r\right)^{-2 d k} \leq \beta^{-t k(2 d+1)} .
$$

This is impossible if $t \rightarrow \infty$.

Let $M^{n}$ be a complete noncompact manifold, for $0 \leq q \leq n$ and for $d>0$, let

$$
\mathcal{H}_{d}^{q}(M)=\left\{u \mid u \text { is a harmonic } q \text { form, and }|u|(x) \leq C(1+r(x))^{d} \text {, for some } C\right\} .
$$

In case $q=0$, that is, in the case of harmonic functions, we simply write $\mathcal{H}_{d}(M)$ instead of $\mathcal{H}_{d}^{0}(M)$. Using Lemmas 1.1 and 1.3, as in [L 1], we have 
Theorem 1.4. Let $M^{n}$ be a complete manifold such that Ric $(x) \geq-K(1+r(x))^{-2}$, for some constant $K$, where $r(x)$ is the distance of $x$ from $p$. Suppose that each end of $M$ with respect with $B_{p}(1)$ satisfies (VC) . Then there is a constant $C$ independent of $d$ such that

$$
\operatorname{dim} \mathcal{H}_{d}(M) \leq C d^{n-1} .
$$

Proof. Note that if $u \in \mathcal{H}_{d}(M)$, then $\Delta u^{2} \geq 0$. Let $\mathcal{K}$ be a $k$-dimensional subspace of $\mathcal{H}_{d}(M)$. We may assume that $d \geq 1$. Let $1>\delta>0$, and let $\epsilon=\frac{1}{5 d}$. By Lemma 1.1, there is a constant $C_{1}$ independent of $d$ and $\delta$, and there is $r_{0}>0$ which may depend on $\delta$ such that if $r>r_{0}$, and if $\left\{u_{1}, \ldots, u_{k}\right\}$ is an orthonormal basis for $\left(\mathcal{K}, S_{(1+\epsilon) r, 1}\right)$, then

$$
\sum_{i=1}^{k} S_{r, 1}\left(u_{i}, u_{i}\right) \leq C_{1} \epsilon^{-n-\delta+1} .
$$

On the other hand by Lemma 1.3 , we can find $r>r_{0}$ so that if $\left\{u_{1}, \ldots, u_{k}\right\}$ is an orthonormal basis for $\left(\mathcal{K}, S_{(1+\epsilon) r, 1}\right)$, then

$$
\sum_{i=1}^{k} S_{r, 1}\left(u_{i}, u_{i}\right) \geq k(1+\epsilon)^{-(2 d+1)} .
$$

Hence

$$
\begin{aligned}
k & \leq C_{1}(1+\epsilon)^{(2 d+1)} \epsilon^{-n-\delta+1} \\
& =C_{1}\left(1+\frac{1}{5 d}\right)^{2 d+1}(5 d)^{n+\delta-1} \\
& \leq C_{2} d^{n+\delta-1}
\end{aligned}
$$

for some constant $C_{2}$ independent of $d$ and $\delta$. Hence

$$
\operatorname{dim} \mathcal{H}_{d}(M) \leq C_{2} d^{n+\delta-1} .
$$

Let $\delta \rightarrow 0$; the result follows.

Similarly, we can prove

Theorem 1.5. Suppose that the Ricci curvature of $M^{n}, n \geq 3$, satisfies Ric( $\left.x\right) \geq$ $-\lambda(r(x))$ where $\lambda:[0, \infty) \rightarrow[0, \infty)$ is a nonincreasing function such that

$$
\int_{0}^{\infty} r^{n-1} \lambda(r) d r<\infty
$$

Suppose also that each end of $M$ with respect to $B_{p}(1)$ satisfies $(\mathbf{V C})$. Then

$$
\operatorname{dim} \mathcal{H}_{d}^{1}(M) \leq C d^{n-1} .
$$

If we assume that the curvature operator at a point $x$ is no less than $-\lambda(r(x))$, then for all $q$, we also have

$$
\operatorname{dim} \mathcal{H}_{d}^{q}(M) \leq C d^{n-1}
$$

Here $C$ is a constant independent of $d$.

Proof. By assumptions on the Ricci curvature, we have

$$
\Delta|u|^{2} \geq-C_{1} \lambda|u|^{2},
$$

for all harmonic one forms. If in addition, the assumption on the curvature operator is also satisfied, then the above inequality is also true for any harmonic forms. Using 
Lemma 1.2 instead of Lemma 1.1, the proof is similar to the proof of Theorem 1.4 .

Remark 1.6. The following are some examples of manifolds $M$ satisfying the assumptions of Theorem 1.4:

1. $M$ has nonnegative sectional curvature outside a compact set, or more generally, the sectional curvature satisfies $K_{M}(x) \geq-\lambda(r(x))$, where $\lambda$ is a nonnegative, nonincreasing function on $[0, \infty)$ such that $\int_{0}^{\infty} r \lambda(r) d r<\infty$ [L-T 5].

2. $M^{n}$ has nonnegative Ricci curvature outside $B_{p}(1)$, Ric $\geq-\epsilon(n)$, where $\epsilon(n)$ is a small number depending only on $n$, and if $M$ has two ends, [L-T 5].

3. $M$ had nonnegative Ricci curvature outside a compact set, with finite first Betti number. In this case, even though it is not exactly true that each end of $M$ satisfies (VC), however, it is almost true so that by modifying some arguments of Lemma 1.1, Theorem 1.4 still holds for such manifolds; see [L-T 5, Corollary 6.2] in particular.

\section{HARMONIC FORMS ON COMPLETE SURFACES}

In Theorem 1.5 we assume that the dimension of the manifold is at least 3 . In case $M$ is a surface, and if $M$ satisfies the curvature assumption in the theorem, then $M$ will have finite total curvature; see [Hu]. Hence it would be interesting to understand the dimension of the space of polynomial growth harmonic forms on such a surface. The case of harmonic functions ( 0 -forms), and hence harmonic two forms, have been studied in [L-T 2]. Only the case for harmonic 1-forms remains to be studied. Let $M$ be a complete noncompact surface with finite total curvature, and let $p \in M$ be a fixed point. Then $M$ is conformally equivalent with a compact Riemann surface of genus $g$ with finitely many points deleted $[\mathrm{Hu}]$. Let $g$ be the genus of the surface, and $l$ be the number of points deleted. Let $E_{i}, 1 \leq i \leq l$, be the ends of $M$, and let

$$
\alpha_{i}=\lim _{r \rightarrow \infty} \frac{A\left(B_{p}(r) \cap E_{i}\right)}{\pi r^{2}}
$$

which exists by [Ha]. Denote the dimension of the space of harmonic polynomials of degree less than or equal to $k$ with zero constant term in $\mathbb{R}^{2}$ by $N_{k}$. If $k<0$, then $N_{k}=0$ by convention.

Lemma 2.1. Let $M$ be a complete surface of finite total curvature. Let $\mathcal{H}_{d}^{c l}(M)$ be the space of closed harmonic 1-forms with polynomial growth of degree at most $d$. Then

$$
\operatorname{dim} \mathcal{H}_{d}^{c l}(M) \leq \sum_{i=1}^{l} N_{\alpha_{i}(d+1)}+2(g+l)-1
$$

Proof. Since the singular homology class $H_{1}(M ; \mathbb{R})$ is a vector space of dimension $2 g+l-1$,

$$
\operatorname{dim} \mathcal{H}_{d}^{c l}(M) \leq 2 g+l-1+\operatorname{dim}\left(\mathcal{H}_{d}^{c l}(M) \cap\{\text { exact 1-forms }\}\right) .
$$

Let $u \in \mathcal{H}_{d}^{c l}(M) \cap\{$ exact 1-forms $\}$. Then $d u=0$. Since $u$ is harmonic, $d \delta u=0$, and $\delta u$ is a constant function. Hence we can define a linear map

$$
\Phi: \mathcal{H}_{d}^{c l}(M) \cap\{\text { exact } 1 \text {-forms }\} \rightarrow \mathbb{R},
$$


such that $u$ is mapped into $\delta u$. The kernel of this map consists of harmonic 1-forms $u$ on $M$ which is both closed and co-closed with polynomial growth of degree at most $d$. In fact, $u$ is exact. Hence $u=d f$ for some function $f$. We normalize $f$ so that $f(p)=0$. In this case, $f$ is uniquely determined. Since $\delta u=0, f$ is harmonic. Now $u \in \mathcal{H}_{d}^{c l}(M)$ implies

$$
|u|(x) \leq C(1+r(x))^{d}
$$

for some $C$. So

$$
|f|(x) \leq C(1+r(x))^{d+1}
$$

By [L-T 2], we have

$$
\operatorname{dim}(\operatorname{ker}(\Phi)) \leq \sum_{i=1}^{l} N_{\alpha_{i}(d+1)}+l-1
$$

So

$$
\operatorname{dim}\left(\mathcal{H}_{d}^{c l}(M) \cap\{\text { exact 1-forms }\}\right) \leq \sum_{i=1}^{l} N_{\alpha_{i}(d+1)}+l .
$$

From (2.1) and (2.2), the lemma follows.

Lemma 2.2. With the same assumptions and notations in Lemma 2.1, we have

$$
\operatorname{dim}\left\{d u \mid u \in \mathcal{H}_{d}^{1}(M)\right\} \leq \sum_{i=1}^{l} N_{\alpha_{i}(d-1)}+l .
$$

Proof. Note that

$$
\operatorname{dim}\left\{d u \mid u \in \mathcal{H}_{d}^{1}(M)\right\}=\operatorname{dim}\left\{* d u \mid u \in \mathcal{H}_{d}^{1}(M)\right\} .
$$

Let $u \in \mathcal{H}_{d}^{1}(M)$; then $* d u$ is a harmonic function, where $*$ is the usual star operator. For any $R>0$, let $\phi \geq 0$ be a smooth function with compact support in $B_{p}(4 R)$, such that $\phi=1$ in $B_{p}(2 R)$, and $|d \phi| \leq \frac{2}{R}$. Then

$$
\begin{aligned}
0 & =\int_{M}\left\langle(d \delta+\delta d) u, \phi^{2} u\right\rangle \\
& =\int_{M}\left(\left\langle\delta u, \delta\left(\phi^{2} u\right)\right\rangle+\left\langle d u, d\left(\phi^{2} u\right)\right\rangle\right) \\
& =\int_{M} \phi^{2}\left(|\delta u|^{2}+|d u|^{2}\right)-\int_{M} \delta u\left\langle d\left(\phi^{2}\right), u\right\rangle+\int_{M}\left\langle d u, d\left(\phi^{2}\right) u\right\rangle,
\end{aligned}
$$

where $\delta$ is the formal adjoint of $d$. By Schwartz inequality, we have

$$
\int_{M} \phi^{2}\left(|\delta u|^{2}+|d u|^{2}\right) \leq 2 \int_{M}|d \phi|^{2}|u|^{2}
$$

Hence

$$
\int_{B_{p}(2 R)}|d u|^{2} \leq \frac{4}{R^{2}} \int_{B_{p}(4 R)}|u|^{2}
$$

Let $f=* d u$; then

$$
\int_{B_{p}(2 R)} f^{2} \leq \frac{4}{R^{2}} \int_{B_{p}(4 R)}|u|^{2}
$$


By the assumption on $u$, and the fact that the volume growth of $M$ is at most quadratic, see [Ha], there is a constant $C_{1}$, such that

$$
\int_{B_{p}(2 R)} f^{2} \leq C_{1}\left(1+R^{2 d}\right) .
$$

Let $E_{i}$ be an end. We may assume that $E_{i}$ is conformally equivalent to the exterior of the unit disk in the complex plane. We may assume that the metric is extended conformally and smoothly into the unit disk. Let $o$ be the origin; by (2.3), we conclude that

$$
\int_{B_{o}(2 R)} f^{2} \leq C_{1}\left(1+R^{2 d}\right) .
$$

Since $f$ is harmonic outside the Euclidean unit disk, using cut off function, it is not hard to prove

$$
\int_{B_{o}(R)}|\nabla f|^{2} \leq C_{2}\left(1+R^{2 d-2}\right)
$$

for some constant $C_{2}$ which is independent of $R$. Let $R_{0}$ be the largest $\rho$ such that the Euclidean disk with center at $o$ and radius $\rho$ lies inside $B_{o}(R)$. In this case, there is a point $x \in \partial B_{o}(R)$, such that the Euclidean distance of $x$ from $o$ is $R_{0}$. By [L-T 2], for all $\epsilon>0$,

$$
R \leq\left(R_{0}\right)^{\alpha_{i}+\epsilon}
$$

if $R$ is large enough. Hence by (2.4),

$$
\int_{\tilde{B}_{o}\left(R_{0}\right)}\left|\nabla_{0} f\right|^{2} \leq C_{4}\left(1+R_{0}^{2(d-1)\left(\alpha_{i}+\epsilon\right)}\right)
$$

for some constant $C_{4}$, where $\tilde{B}_{o}(r)$ is the Euclidean disk of radius $r$ with center at $o$, and $\nabla_{0}$ is the Euclidean gradient of $f$. By the mean value inequality in $\mathbb{R}^{2}$, if $r(x)$ is large then

$$
\left|\nabla_{0} f(x)\right| \leq C_{5}\left(r_{0}^{-1}(x)+r_{0}(x)^{(d-1)\left(\alpha_{i}+\epsilon\right)-1}\right)
$$

where $r_{0}(x)$ is the Euclidean distance of $x$ from $o$. By the method in [L-T 2], we conclude that

$$
f(x)=f_{i}(x)+f_{i}^{*}(x)+\beta_{i} \log r_{0}(x)
$$

near infinity of $E_{i}$, where $f_{i}$ is a harmonic polynomial on $\mathbb{R}^{2}$ with degree not greater than $(d-1)\left(\alpha_{i}+\epsilon\right)$ with zero constant term, $\beta_{i}$ is a constant, and $f_{i}^{*}$ is a bounded harmonic functions. As in [L-T 2], we can conclude that

$$
\operatorname{dim}\left\{d u \mid u \in \mathcal{H}_{d}^{1}(M)\right\} \leq \sum_{i=1}^{l} N_{\alpha_{i}(d-1)}+l .
$$

Combining Lemmas 2.1 and 2.2, we have: 
Theorem 2.3. Let $M$ be a complete surface with finite total curvature. Using the notations as in Lemma 2.1, we have

$$
\operatorname{dim} \mathcal{H}_{d}^{1}(M) \leq \sum_{i=1}^{l}\left(N_{\alpha_{i}(d+1)}+N_{\alpha_{i}(d-1)}\right)+2 g+3 l-1 .
$$

\section{REFERENCES}

[B-C] R. Bishop and R. Crittenden, Geometry of Manifolds, Academic Press: New York, 1964. MR 29:6401

[Cg-Y] S. Y. Cheng and S. T. Yau, Differential equations on Riemannian manifolds and their geometric applications, Comm. Pure Appl. Math. 28 (1975), 333-354. MR 52:6608

[C-M 1] T. Colding and W. Minicozzi, On function theory on spaces with a lower Ricci curvature bound, Math. Research Letters 3 (1996), 241-246. MR 97e:53073

[C-M 2] T. Colding and W. Minicozzi, Harmonic functions with polynomial growth, preprint.

[C-M 3] T. Colding and W. Minicozzi, Large scale behavior of kernels of Schrödinger operators, preprint.

[C-M 4] T. Colding and W. Minicozzi, Generalized Liouville properties for manifolds, Math. Res. Lett. 3 (1996), 723-729. MR 97h:53039

[C-M 5] T. Colding and W. Minicozzi, Harmonic functions on manifolds, preprint.

[C-M 6] T. Colding and W. Minicozzi, Liouville theorems for harmonic sections and applications manifolds, preprint.

[C-M 7] T. Colding and W. Minicozzi, Weyl type bounds for harmonic functions, preprint.

[G] A. Grigor'yan, The heat equation on noncompact Riemannian manifolds, Math. USSR Sbornik. 72 (1992), 47-77.

[Ha] P. Hartman, Geodesic parallel coordinates in the large, Amer. J. Math. 86 (1964), 705727. MR 30:3435

$[\mathrm{Hu}$ A. Huber, On subharmonic functions and differential geometry in the large, Commentarii Math. Helv. 32 (1957), 13-72. MR 20:970

[L 1] P. Li, Polynomial growth harmonic sections, to appear in Math. Res. Letters.

[L 2] P. Li, Curvature and function theory of Riemannian manifolds, preprint.

[L-S] P. Li and R. Schoen, $L^{p}$ and mean value properties of subharmonic functions on Riemannian manifolds, Acta Math. 153 (1984), 279-301. MR 86j:58147

[L-T 1] P. Li and L. F. Tam, Linear growth harmonic functions on a complete manifold, J. Diff. Geom. 29 (1989), 421-425. MR 90a:58202

[L-T 2] P. Li and L. F. Tam, Complete surfaces with finite total curvature, J. Diff. Geom. 33 (1991), 139-168. MR 92e:53051

[L-T 3] P. Li and L. F. Tam, The heat equation and harmonic maps of complete manifolds, Invent. Math. 105 (1991), 1-46. MR 93e:58039

[L-T 4] P. Li and L.-F. Tam, Harmonic functions and the structure of complete manifolds, J. Differential Geom. 35 (1992), 359-383. MR 93b:53033

[L-T 5] P. Li and L.-F. Tam, Green's functions, harmonic functions and volume comparison, J. Differential Geom. 41 (1995), 277-318. MR 96f:53054

[SC] L. Saloff-Coste, A note on Poincaré, Sobolev, and Harnack inequalities, Inter. Math. Research Notices 2 (1992), 27-38. MR 93d:58158

[W] J. Wang, Linear growth harmonic functions on complete manifolds, Comm. Anal. Geom. 4 (1995), 683-698. MR 96m:53044

[Y] S. T. Yau, Harmonic functions on complete Riemannian manifolds, Comm. Pure Appl. Math. 28 (1975), 201-228. MR 55:4042

Department of Mathematics, The Chinese University of Hong Kong, Shatin, Hong KONG

E-mail address: lftam@math.cuhk.edu.hk 